\title{
Rites d'interaction et management en Chine : Le cas de la province du Guangxi
}

\author{
Laurent MERIADE ${ }^{1}$, Li YIQIANG ${ }^{2}$
}

\begin{abstract}
Résumé
Dans la culture chinoise (comme probablement dans d'autres cultures), les valeurs religieuses se sont construites à partir de croyances plus anciennes souvent afin de réduire l'influence du polythéisme et des cultes païens. Ces croyances populaires se fédèrent autour de l'enseignement et de la mise en pratique de valeurs morales et spirituelles présentées sous la forme de rites d'interaction par la littérature classique chinoise puis par les principales religions (Confucianisme et Taoïsme notamment).

Nous proposons ici, à travers l'étude de cas d'un groupe d'État chinois adossée à une analyse des valeurs culturelles et religieuses retranscrites dans des rites individuels et collectifs, de mettre en évidence la complexité et l'interdépendance des valeurs et croyances qui guident les attitudes et comportements managériaux.

Il semble, comme le démontre notre étude, que la ritualisation du management en Chine soit assez significativement explicitée par les managers qui font une description de valeurs et principes d'action assez proches de ceux contenus dans les rites d'interaction sociale définis par les principaux écrits religieux ou culturels.

Pour mieux appréhender les liens entre les rites culturels d'interaction sociale et les pratiques managériales en Chine, nous mobilisons l'approche des rites d'interaction proposée par Goffman (1974) et reprise par Collins (2004) qui précise le contenu et les conséquences de ces rites sur le comportement des membres d'un groupe ou d'une communauté.

En s'efforçant de réaliser une description vitaliste et culturaliste (Pesqueux et Martinet, 2013) du management chinois, nous cherchons, sur le plan théorique, à déterminer de quelles manières des rites culturels d'interaction sociale peuvent participer à la construction de rituels managériaux dans les organisations. Ceci s'inscrit dans une volonté de contribution aux débats initiés en Occident sur l'éventuel développement d'une théorie du management chinois (Barney et Zhang, 2009) en essayant d'identifier le rôle des rites d'interaction issus de la culture traditionnelle chinoise dans l'application en Chine d'outils développés ailleurs.

Pour cela, sur le plan pratique, nous souhaitons décrire les rites d'interaction d'origine culturelle qui déterminent notablement les comportements managériaux en Chine et génèrent certaines régularités pouvant prendre la forme de rites d'interaction au sens de Collins (2004) et Goffman (1974).

Pour rendre compte de ces éventuelles régularités managériales, nous testons, sur le plan méthodologique, l'analyse quantitative et qualitative d'une enquête réalisée auprès des managers d'un groupe d'État chinois afin de mesurer sa capacité à dépasser certains biais mis en avant dans la littérature (Adler et al., 2009).
\end{abstract}

Mots clés : management, croyances, religions, rites, confucianisme, Chine.

1 Maitre de conférences HDR en Sciences de Gestion - Université Clermont Auvergne - IAE Clermont Auvergne - France

2 Guangxi University of Finance and Economics - 530003 Nanning - Guangxi - China 


\begin{abstract}
In Chinese culture (as probably in other cultures), religious values are built from older beliefs often to reduce the influence of polytheism and pagan cults. These popular beliefs unite around the teaching and practice of moral and spiritual values presented in the form of interaction ritual by classical Chinese literature and by the major religions (Confucianism and Taoism in particular).

We propose, through the case study of a Chinese state group backed by analysis of cultural and religious values transcribed in individual and collective rites, highlighting the complexity and interdependence of values and beliefs that guide managerial attitudes and behaviors.

It seems, as our study shows it, that the ritual management in China is quite significantly explained by managers who make a description of values and principles of action quite close to those found in social interaction rituals defined by the main religious or cultural writings.

To better understand the links between cultural rituals of social interaction and managerial practices in China, we mobilize the ritual approach proposed by Goffman (1974) and adopted by Collins (2004) which specifies the content and consequences of rituals on the behavior of members of a group or a community.

In striving to achieve a vitalistic and culturalist description (Pesqueux and Martinet, 2013) of Chinese management, we seek theoretically, to determine in what ways the cultural rituals of social interaction can participate in the construction of managerial rituals in organizations. This is part of a willingness to contribute to debates initiated in the West about the possible development of a theory of Chinese management (Barney and Zhang, 2009) trying to identify the role of interaction ritual from traditional culture Chinese in China in the application of tools developed elsewhere.

To do this, in practice, we want to describe the interaction ritual of cultural origin that determine managerial behavior significantly in China and generate certain regularities that may take the form of rituals of interaction within the meaning of Collins (2004) and Goffman (1974).

To account for these potential managerial patterns, we test, methodological, quantitative and qualitative analysis of a survey on managers of Chinese State Group to measure its ability to overcome certain bias set before in the literature (Adler et al. 2009).
\end{abstract}

Key words: management, rites, beliefs, religions, confucianism, China. 


\section{Introduction}

Les entreprises tendent avec la mondialisation à se situer au centre d'expressions religieuses multiples qui ne sont pas sans influence sur leurs modes de fonctionnement et de management. Ceci fait apparaitre de nouvelles contingences managériales qui sont paradoxalement assez peu étudiées de manière directe par les chercheurs en gestion. La place de cette expression religieuse dans les organisations est pourtant centrale dans la mesure où elle interroge la capacité des entreprises à rechercher un équilibre entre l'absolutisme religieux et le déni total des croyances qui s'y rapportent (Lilla, 2010).

Les religions qui bâtissent des doctrines et des dogmes à partir d'un ensemble de croyances présentent de fortes spécificités sociale, cognitive et organisationnelle. A partir des doctrines religieuses auxquelles ils adhèrent, leurs pratiquants ou leurs croyants présentent souvent des schèmes interprétatifs extrêmement homogènes (Bartunek, 1984). Cela a une conséquence intéressante pour les techniques de management puisque cela permet de qualifier un certain nombre de comportements à partir de valeurs communes qui à la fois orientent les actions managériales mais aussi peuvent les confronter à des valeurs connexes, concurrentes ou encore opposées.

L'objectif général de ce travail de recherche est de déterminer comment des rites d'interaction culturels et religieux peuvent influencer les comportements managériaux en Chine.

Scheid (1985) rappelle le sens de religieux (religio = à la fois attachement et scrupuleux en latin) qui suppose l'exécution minutieuse de rites. Pour Benveniste (1969), la religion est à la fois lien (religare = relier) et relecture (religere $=$ se relire), activités pour lesquelles les rites sont déterminants car, en se situant entre l'automatique et le réflexif (Dumézil, 1966), ils se distinguent des activités ordinaires en refusant l'improvisation ou l'automatisation des comportements (Héran, 1986).

L'intérêt managérial des études connectant management et religions réside dans la description des pratiques, des convictions ou de croyances qui influencent directement ou indirectement les comportements professionnels et plus particulièrement ceux des managers.

Dans le cas de la culture chinoise (comme probablement dans d'autres cultures), les valeurs rattachées aux religions officielles (confucianisme, taoïsme ou bouddhisme) se sont construites à partir de croyances plus anciennes afin de réduire l'influence du polythéisme et des cultes païens. Les croyances sont nonexclusives voire syncrétiques ce qui offre une place essentielle à un système de rites d'interaction qui s'est construit dans la culture chinoise par sédimentation de croyances provenant de divers cultes.

En occident, les rites sont perçus comme des activités cérémonielles pratiquées dans une religion (Littré). Plus que des pratiques religieuses ou cérémonielles ostensibles et au demeurant persistantes dans la société chinoise, les rites sont surtout des rites d'interaction sociale sans véritable discours théologique 
(Granet, 1968). Et leur reconnaissance passe par leur intériorisation par l'ensemble du corps social afin de relier ses membres et construire les conditions de l'harmonie d'un groupe. Nous souhaitons nous concentrer sur ces rites d'interaction car dans le cas de la Chine, même s'ils se retrouvent dans les traditions religieuses, ils sont avant tout culturels et disposent d'une influence plus large sur les comportements que les rites religieux essentiellement réservés à l'espace privé.

De nombreuses analyses relatives aux relations entre culture et management en Chine mobilisent principalement les cadres d'analyse du confucianisme (Xing,1995; Fan 2000) et à un degré moindre ceux du taoïsme ou du bouddhisme (Fang, 2012). Plus récemment, certains auteurs ont élargi les approches culturelles du management en Chine en insistant sur la grande diversité des influences culturelles en décrivant la forte emprise de « l'art de la guerre » ou du « légalisme » sur les consciences chinoises (Pan et al, 2012).

La multiplicité des interprétations culturelles du management en Chine rend son analyse délicate alors que nombre de ces interprétations mobilisent des éléments de la culture traditionnelle chinoise repris par la suite dans les écrits religieux. Par contre, peu de travaux insistent sur la place des rites d'interaction sociale compilés dans les livres classiques de la culture chinoise et qui pourtant retranscrivent les principales caractéristiques culturelles des chinois.

Goffman (1974) a développé une approche du rite à travers le concept de rituel qui dépasse les références au sacré et au magique du rite pour décrire les interactions que ce dernier construit et qui façonnent la vie sociale (Goffman, 1974). Il sort le rite de son spectre purement religieux pour le diffuser dans la « vie séculière » et en élargit sa lecture à ses dimensions sociales et interactionnistes. Pour illustrer cette élargissement lexical, il utilise le concept de rituel défini comme une attitude imposée par une société à ses membres et qui «s'exprime par un mode de comportement traditionnel» (Goffman, 1974, p. 51). A la suite de Goffman, Collins (2004) propose de rechercher dans les interactions humaines un processus de ritualisation (Interaction ritual chain) dans lequel s'agrègent des émotions et humeurs communes à un groupe ou une culture. Reprenant les conséquences d'un rituel (solidarité de groupe, énergie émotionnelle, symboles d'appartenance et règles communes) définis par Durkheim (1995), il ouvre une perspective féconde pour les travaux en management en proposant un modèle descriptif des éléments représentatifs d'une ritualisation des comportements.

Sur le plan théorique, en proposant une description vitaliste et culturaliste (Pesqueux et Martinet, 2013) des comportements des managers chinois, nous cherchons à déterminer de quelles manières des rites culturels d'interaction sociale peuvent participer à la construction de rituels managériaux dans les organisations. 
Sur le plan pratique, nous souhaitons décrire les rites d'interaction d'origine culturelle qui déterminent notablement les comportements managériaux en Chine et génèrent certaines régularités pouvant prendre la forme de rites d'interaction au sens de Collins (2004) et Goffman (1974).

Sur le plan méthodologique, à une analyse en composante principale (Pan et al., 2012) des réponses à une enquête réalisée auprès des managers d'un groupe d'État chinois, nous associons une analyse qualitative afin afin de mesurer la capacité de cette méthodologie à dépasser certains biais mis en avant dans la littérature (Adler et al., 2009).

\section{Management et religions dans la littérature}

\subsection{Les approches culturelles}

Comme le souligne d'Iribarne (2011), la culture est « un référentiel de sens » pour les conduites humaines dans les organisations. Les liens entre religions et management ne peuvent pas être appréciées dans l'absolu, sans tenir compte de l'époque et du lieu. Au-delà d'éléments objectifs proches des enjeux publics anciens et modernes, les comportements managériaux dépendent aussi de données culturelles qui déterminent les attitudes, les manières d'agir, les droits et les devoirs de chacun. Sans vouloir faire abstraction des critiques portant sur l'essentialisme des approches « culturalistes » (Pesqueux, 2010), nous considérons, à la suite de notamment Cazal et al. (2010), que les comportements et les choix managériaux sont influencés par les valeurs d'une culture nationale qui, sans être monolithiques et stables, sont «conditionnantes pour les pratiques et conditionnées par ces pratiques». Des comportements qui, dans l'organisation, sont l'expression de l'hétérogénéité de parcours et de valeurs personnelles (Sainsaulieu, 1988), eux-mêmes conditionnés par des éléments culturels nationaux.

Nous parlons ici d'approche « culturaliste » pour circonscrire des éléments descriptifs des rites culturels en Chine (schèmes cognitifs, valeurs et attitudes des individus et des collectifs) qui expliquent et donnent du sens aux valeurs professionnelles. La culture est ainsi observée comme « une construction sociale en constant renouvellement » (Cuche, 2004).

Un certain nombre de travaux ont été réalisés sur le thème des comportements professionnels des chinois afin de présenter leurs particularismes et leurs effets dans les interactions humaines. Ces travaux présentent les principes qui gouvernent leurs conduites dans des situations professionnelles ou commerciales (Faure et Chen, 1997; Lin, 1997; Fernandez et Zheng, 2008; Goxe, 2012). C'est dans ces études que l'on retrouve les références les plus importantes au respect de la hiérarchie en Chine, aux obligations vis à vis du groupe et de la famille, à la nécessité de ne pas perdre la « face » notamment dans les relations professionnelles (Faure, 2004; Lavin, 1994; Wang, 1981).

D'autres travaux encore plus analytiques portent sur les valeurs des Chinois, tels que le système 
d'obligations mutuelles, le rôle du réseau relationnel, la gestion du temps, le rôle de l'harmonie, les tactiques pour se venger, etc. (Knutsson, 1986; Brunner et Wang, 1988; Faure, 2003). Ces travaux utilisent le confucianisme comme principal cadre de compréhension et de descriptif de ces valeurs chinoises. Yau (2004) ou encore Pan et al. (2012) sans réfuter l'utilisation de ce cadre d'analyse confucéen tente de le perfectionner en y agrégeant des analyses mobilisant d'autres traditions philosophiques (Taoisme, Bouddhisme, Légalisme ou Art de la guerre).

Le rôle occupé par des vertus telles que la patience, l'harmonie, le respect de la hiérarchie, le réseau social (le guanxi) ont été très tôt mis en avant pour relier des croyances religieuses et des comportements sociaux (Yang, 1957; Bruner et al., 1989).

A la suite de Levi (2003), Faure (2003) décrit les six valeurs fondamentales du confucianisme comme des éléments expliquant l'essentiel des comportements managériaux en Chine : le "Ren » (le sens de l'humain), le «Yi » (l'équité rituelle), le « $L i$ » (les rites), le «De» (la morale, conformité aux règles), le « Xin » (la confiance) et le «Zhi» (la connaissance des choses et des hommes), chaque composante s'articulant avec les autres et reposant sur les autres.

Goxe (2012) retient lui aussi quatre éléments caractéristiques du management chinois qui se retrouvent inscrits dans la pensée confucéenne : l'orientation collective (vers le groupe et notamment vers la famille), le respect de la hiérarchie et de l'âge, l'importance des relations interpersonnelles (guanxi) et le concept de « face ».

Ams (2008) puis Ascencio et Rey (2010) reprennent ces descriptions pour définir les fondamentaux de la négociation et du management en Chine autour de l'importance du guanxi, de la logique de «face » et de la culture morale, valeurs qu'ils rattachent explicitement au confucianisme.

Réfutant quelque peu cette profusion d'examens de la culture managériale chinoise, Kulich et Zhang (2010, p. 248) retrouvent dans leur revue de littérature vingt-trois valeurs culturelles chinoises essentielles (yuan, jia, guanxi, mianzi, lian, etc.) nées du syncrétisme de la pensée chinoise et ne pouvant pas être rattachées à une tradition philosophique particulière. Ce syncrétisme des valeurs culturelles chinoises se retrouve dans les rites d'interaction développés dans la culture traditionnelle et repris par la suite dans les écrits philosophiques et religieux.

\subsection{Les rites : une empreinte essentielle des comportements managériaux}

Pour Durkheim (1894) le rite prend la forme d'une habitude collective, qui peut s'exprimer dans la tradition orale comme écrite et à laquelle on peut attribuer une force morale tout aussi puissante que celle attribuée aux règles juridiques ou morales. A l'image des propos tenus par Mauss (1947, p. 241) qui insiste 
sur la complémentarité des rites oraux et des rites ostentatoires, Goffman (1974) puis Collins (2004) construisent la définition des rites d'interaction à partir de la tradition anthropologique et des " rites interpersonnels » définis par Durkheim (1894).

En matière de management, Weber (1921), dans sa description des principes bureaucratiques, décrit le rite comme un élément de rationalisation car il attribue au leadership et aux procédures bureaucratiques une légitimité institutionnelle en construisant des routines et des habitudes de travail respectées. Même s'il est très significativement opposé à la conception «wéberienne » du rite, Pareto (1917), tout en défendant la thèse de l'irrationalité du rite, admet que celui-ci vaut essentiellement par sa capacité à relier les hommes et les idées. Ainsi, pour Weber (1917) comme pour Pareto (1917), la théorie de l'action est avant tout une théorie de l'action rituelle qui fixe des procédures de travail qui en se déconnectant des individus œuvre pour la rationalisation du management (Weber, 1921). Le rite en tant que principe organisateur de pratiques recherche une capitalisation collective mais dont l'initiative est individuelle. Ces conceptions du rite sont à rapprocher du rite d'interaction tel que le définit Goffman (1974) car elles permettent de circonscrire le rite dans ses dimensions à la fois cérémonielles mais surtout sociales.

Déjà Fustel de Coulanges (1864) précisait que si la vie rituelle s'objective avant tout par son formalisme, elle se mesure dans chacun des êtres par la croyance dans le lien social qu'elle entretient de génération en génération. Par contre, pour Fustel, l'apparition du monothéisme chrétien correspond à la « déritualisation » progressive de la société occidentale.

En Chine, cette «déritualisation» de la société ne semble jamais s'être exécutée que ce soit par le truchement d'une éventuelle concurrence religieuse ou par la volonté des souverains. Au contraire, dans la construction des différentes dynasties impériales puis de l'État chinois, les rites ont supplanté et pris la suite des pratiques religieuses car ils offraient l'opportunité d'unifier et de transcender un ensemble de cultes d'origines et d'époques diverses.

Aujourd'hui, pour définir ces rites, plutôt que de parler uniquement de pratiques ou de cérémonies religieuses, Granet décrit un « corps de conventions séculaires dont l'observance libère la vie de risques indéfinis » (1968, p. 163) qui agrègent un ensemble de croyances à vocation morale et éthique. La reconnaissance de ces rites passe par leur intériorisation par l'ensemble du corps social afin de relier ses membres et construire les conditions de l'harmonie d'un groupe.

Romar (2004) suggère ainsi que les rites culturels sont à la fois éloignés et compatibles avec un grand nombre de préceptes de gestion occidentaux à la condition de les percevoir dans leurs dimensions dynamiques recherchant une harmonie des idées et des pratiques. Il en est ainsi du respect du "guanxi» (le réseau social de chaque individu) qui est à la base de l'ordre social confucéen (Kulich et Zhang, 2010) 
et également fortement compatible avec les concepts managériaux occidentaux de management participatif(Drucker, 1954) ou collaboratif (Drucker, 1993).

Ces pratiques rituelles (guanxi, face, piété filiale, centralité notamment) ont pour vocation de nouer un lien symbolique entre des croyances diverses pour construire les limites d'une communauté de pensée qui n'a pas de vocation purement religieuse mais plutôt une fonction sociale au sein de laquelle les managers ou les cadres vont détenir un rôle à la fois de médiation et d'exemplarité (Kulich et Zhang, 2010). Pour définir l'influence de ces rites sur les comportements professionnels, il est difficile de parler de déterminisme religieux (Weber, 1917) mais plutôt d'un système de pensée agrégeant des croyances hétérogènes qui se manifestent autour de valeurs morales fondamentales. Par sa culture, l'homme est alors une sorte de construction élaborée à partir de ces règles morales (Goffman, 1974) qui se retrouvent dans le suivi et le respect de rites d'interaction sociale.

Pour Granet (1922, p. 164), dans la société chinoise, « tout rite peut être profitable ; aucune croyance n'a d'intérêt véritable » et c'est le sens que, dans un premier temps, les souverains ont pu donner aux rites culturels dans les livres classiques et qui ont été diffusé par la suite par les écrits confucéens et taoïstes. Le syncrétisme que représente le suivi de ces rites est une manière de réinterpréter et de rapprocher des traditions religieuses et philosophiques diverses pour leur attribuer une certaine intemporalité dans l'esprit des chinois.

L'histoire de la pensée chinoise est ancestrale et totalement imbriquée dans le développement des mouvements religieux (Cheng, 2007, Goossaert, 2007). Les chinois observaient, bien avant l'apparition du confucianisme, des croyances ancestrales qu'ils n'ont jamais perdues car elles correspondent à des pratiques populaires liées au culte agraire et à celui de la famille qui n'ont pas été infirmées par les religions officielles (confucianisme, taoïsme et bouddhisme).

« Les Chinois ne sont pas des théologiens mais des ritualistes » (Barbier, 2001). Et les thèses développées par Billeter (2006) réfutant le «mythe de l'altérité » ou de la spécificité de la culture chinoise se montrent, selon Gernet (2001), très éloignées du quotidien des chinois, au contraire fortement marqué par le suivi régulier de rites « civils et religieux ». Dans les pratiques courantes des chinois, à l'heure actuelle, nombre de ces rites sont encore très respectés et ils restent profondément ancrés dans les consciences collective et individuelle. Ainsi, pour Yeh et Bedford (2004), le jia (le clan) et le xiao (piété filiale) constituent les bases principales de la réflexivité des salariés chinois dans leurs stratégies de carrière professionnelle alors qu'ils sont à l'origine des rites d'éducation repris par Confucius dans ses « Quatre canoniques ».

L'exercice de ces rites dans les entreprises n'est pas toujours apparent (encore que dans de nombreuses situations le suivi des rites familiaux tels que la piété filiale ou le guanxi l'emporte largement sur les 
impératifs de l'entreprise) par contre ils définissent des modes de pensée et d'action ritualisés.

En Chine, les rites peuvent être vus non comme des pratiques religieuses comme on l'entend en Occident, c'est à dire cérémonielles (même si ils peuvent parfois en prendre l'aspect) mais plutôt comme des règles d'interaction sociale (Goffman, 1974 ; Collins, 2004) Le respect de ces rites permet l'intériorisation de valeurs morales propres à un ensemble culturel reliant préceptes religieux (confucianistes, taoïstes ou encore bouddhistes) et héritages issus des « Cinq classiques » de la littérature traditionnelle. Sous ce nom sont généralement consignés les «Cinq classiques » de la culture traditionnelle chinoise (le yijing ou livre de mutation, le shijing livre de vers, le liji livre des rites, le shujing livre des documents, le chunqiu chronique des printemps et des automnes), appellation donnée à des textes datant du Xe au VIIe siècle avant notre ère et donc antérieurs à Confucius (551 à 479 avant J.-C) qui leur associa des ouvrages de commentaires dénommés les "Quatre canoniques» confucéens (le Lun Yu entretiens de Confucius, le Meng Zi les enseignements de Mencius, le Da Xue les grandes études, et le Zhong Yong l'application de la centralité).

Le Liji (le livre des rites) est assez caractéristique de cette préséance des rites dans la culture chinoise car il rassemble un grand nombre de pratiques morales et vertueuses glorifiées par les dynasties anciennes de la chine et qui seront compilées et commentées par la suite par Confucius. Ce livre des rites fait partie d'un ensemble d'ouvrages écrits pendant la première partie de la dynastie des Zhou (Zhou de l'ouest, 1046-771 av. J.-C.). Il décrit les bienséances et les cérémonies à respecter afin qu'elles intègrent la conscience des hommes et permettent de construire l'harmonie sociale. La civilité, les bonnes manières et les devoirs vis à vis des ancêtres, des parents ou des souverains constituent l'essentiel des rites que la culture classique chinoise recense comme des instruments permettant de canaliser les écarts entre la vie individuelle et la vie collective. Hwang (2011) illustrent les liens étroits entre le respect de rites traditionnels et les interactions humaines à travers la place centrale accordée dans le livre des rites à la de la face (mianzi) et à la moralité (daode).

Ceci rend sans doute spécifiques les formes d'appropriation des outils modernes du management dans les entreprises chinoises et impose une gestion particulière du rapprochement entre modèles de gestion occidentaux et chinois. Ce rapprochement passe à notre sens par une description des valeurs ritualisées par les managers chinois et de leurs relations avec les rites prônés par la culture traditionnelle.

\section{Cadre conceptuel}

Comme nous l'avons signifié plus haut, une importante tradition anthropologique et sociologique s'est concentrée sur la place des rites dans la construction de la société et des interactions humaines (Fustel de Coulanges, 1864; Durkheim, 1894; Mauss, 1950, et plus récemment Héran, 1986). 
S'appuyant sur les résultats de ces travaux, Goffman (1973) soutient que la dimension rituelle se développe au-delà du rite (dont le terme est réservé aux cérémonies) et c'est en cela qu'elle intéresse le chercheur en management. Elle s'étend à toutes les relations sociales visibles ou non entre les membres de groupe et permet la compréhension des habitudes et redondances dans l'exécution de tâches ou choix professionnels.

Collins (2004) approfondit de manière opérationnelle cette approche du rite en proposant une lecture explicative des rites d'interaction sur lesquels les rites culturels ont une influence très significative. Pour les travaux managériaux, le modèle qu'il propose (figure 1), en dynamisant la relation entre les rites observés et les situations vécues, met en valeur l'implication mutuelle de l'acteur et de ses interactions sociales et professionnelles dans la construction de rituels. Collins fait valoir que la situation d'une action n'est pas une construction cognitive en soi mais il la définit comme un processus de partage d'émotions communes et d'intersubjectivité dont les limites s'expriment dans des humeurs et centres d'attention communs (figure 1) déterminés en grande partie par des éléments culturels.

Lorsque deux ou plusieurs personnes se rencontrent dans une situation d'attentions et d'émotions mutuelles, elles peuvent construire un rituel identifiable par quatre conséquences communes (solidarité, énergie émotionnelle, symboles, règles ; figure 1).

Toute organisation peut dès lors être décrite à travers une chaîne de rites d'interaction (Collins 2004) qui se construisent à partir de caractéristiques communes aux membres que «l'effervescence collective »du groupe pourra transcender (figure 1).

\section{Figure 1: Modèle des rites d'interactions (traduit de Collins, 2004, p. 48)}

\section{Caractéristiques des rites d'interaction}

Appartenance à un groupe Barrières à l'entrée

Centres d'attention communs

Humeurs communes

\section{Conséquences des rites d'interaction}

Solidarité de groupe Transmission d'énergie emotionnelle Symboles communs de relations sociales

Règles de moralité

Les rites issus d'une culture et notamment les rites d'interaction sociale détiennent à ce titre tout ou partie de ces caractéristiques ainsi que la capacité de les mobiliser collectivement. C'est pour cela que dans la perspective de se prononcer sur l'existence de rituels managériaux déterminés par des rites culturels d'interaction, nous souhaitons solliciter l'approche de Collins qui offre l'opportunité de décrire une éventuelle ritualisation du management en permettant l'identification de rituels managériaux provenant du 
suivi et du respect de rites encastrés dans une culture.

Nous utilisons l'appellation «rituels » managériaux plutôt que "rites » managériaux pour illustrer la relation de dépendance du rituel managérial vis-à-vis du rite d'interaction sociale d'origine culturelle.

\section{Cadre méthodologique}

Notre approche méthodologique mobilise à la fois des outils quantitatifs et qualitatifs qui sont destinés à définir l'influence des rites d'interaction sociale issus de la culture chinoise sur les comportements managériaux.

Pan et al. (2012) dans leur approche des écoles de pensée chinoises influentes en entreprise proposent une méthodologie identifiant dans un premier temps les idées représentatives de chacune de ses écoles. Ils font appel pour cela à un échantillon de vingt-sept chercheurs issus de diverses disciplines (psychologie, sociologie, littérature et philosophie) pour définir les écoles de pensée chinoises et leurs principales idées.

Afin de construire le contenu de notre enquête (voir Annexe), nous reprenons les résultats de ce travail qui identifient 35 valeurs essentielles de la culture chinoise en les confrontant aux résultats de notre revue de littérature afin de retenir, parmi ces valeurs, celles explicitement issues des "Cinq classiques » ou des «Quatre canoniques » de la culture chinoise qui constituent les principaux supports écrits de la culture traditionnelle chinoise à laquelle nous consacrons notre question de recherche.

Pour construire les questions de notre enquête nous avons retenu les valeurs suivantes qui caractérisent les valeurs issues des livres classiques chinois et que Pan et al. (2012), par leur approche méthodologique, retrouvent dans les écoles de pensée chinoise (l'harmonie, la hiérarchie, la réciprocité, la modération, la qualité des relations humaines, la loyauté, la moralité, le respect des ancêtres, la face, le refus du conflit, l'humilité, le devoir, le respect du réseau social, l'effort).

Notre enquête a consisté à analyser les réponses des cadres et ingénieurs du groupe à une série de questions fermées afin de mettre en avance les rites influençant les comportements des managers chinois dans l'exécution de leur travail. Notre étude porte sur les perceptions des managers du groupe d'Etat Guangxi Beibu Gulf Investment Group Co., Ltd (GBGIG) fondé en Février 2007 dans la Région autonome Zhuang du Guangxi (GGZAR). Ses activités sont commerciale et administrative puisqu'elles ont vocation à développer les échanges entre les entreprises de la province du Guangxi et les pays de l'ASEAN frontaliers notamment le Vietnam, le Laos et le Cambodge à travers la construction d'infrastructures et de zones commerciales et industrielles en mesure d'accueillir des entreprises chinoises ou transfrontalières et d'agglomérer des compétences spécifiques.

Le nombre d'employés est légèrement supérieur à 2000, fin 2013, dont près de $25 \%$ sont des ingénieurs, 
experts ou cadres spécialisés dans la construction des grandes infrastructures telles que les ports, l'approvisionnement en eau, la construction des équipements publics et des routes, le conseil aux entreprises, la conception et le financement de projet, la conception, la fourniture des équipements et des matériaux, la négociation des droits de propriété et de droits de souscription, et de la coopération économique et technologique. Pour cela, GBGIG détient six filiales spécialisées.

Nous avons questionné 153 cadres ou ingénieurs chinois de ce groupe ou de ses filiales (responsables de département, managers commerciaux, cadres RH, directeurs financiers, ingénieurs de production, managers et cadres administratifs) sur un peu plus de 400 cadres que compte le groupe (dont une 250 dans ses filiales).

Ces questionnaires ont été réalisés dans une logique de découverte à partir d'un échantillon restreint de managers mais couvrant l'ensemble des fonctions managériales du groupe (Thietart, 2007). Le choix d'étudier ce groupe d'Etat s'explique par la grande diversité de ses activités (accompagnement et conseil d'entreprises, ingéniérie de production, administration, finance, gestion des ressources humaines, commercialisation) et par la combinaison d'origines surtout publiques des détenteurs des capitaux du groupe mais aussi privées pour deux de ses filiales.

Un échange universitaire et scientifique existant depuis 7 ans entre notre université et celle d'Economie et de Finance du Guangxi à Nanning nous a permis de développer ce travail de recherche et d'accéder à ce groupe et ses filiales. Le retour des questionnaires s'est avéré très difficile à obtenir tant il est délicat de recenser en Chine des informations managériales en dehors de son réseau de connaissances familiales et professionnelles. Pour faciliter la recension, deux tiers des questionnaires ont été administrés en face à face en langue chinoise par les auteurs et grâce à l'intervention de collègues enseignants chercheurs de l'Université du Guangxi. Le recueil des réponses aux 153 questionnaires a été réalisé sur deux ans (2011 et 2012).

Adler et al. (1989), dans leurs travaux, établissent l'incomplétude des analyses factorielles portant sur des variables culturelles en Chine notamment celles déterminées à partir d'une grille de lecture occidentale. Pour tenter de réduire les biais relatifs à la discontinuité des environnements culturels chinois et occidentaux, nous avons appliqué une méthode de recension et d'analyse à partir des compétences des deux auteurs français et chinois dont l'un d'entre eux parle et écrit couramment les deux langues.

Pour réduire les biais liés à la traduction de l'enquête et des réponses obtenues, les opérations de construction des questions, de recension et de dépouillement des réponses ont chaque fois été réalisées simultanément par les deux auteurs de cet article. Le questionnaire a été intégralement traduit en chinois par un des deux auteurs parlant couramment les deux langues. Les réponses obtenues en face à face ont été 
traduites par les deux auteurs français et chinois avec l'aide d'un enregistrement audio. Les réponses obtenues à partir du questionnaire papier ont été traduites et retranscrites sur le logiciel d'analyse par les deux auteurs.

Le questionnaire administré (voir Annexe) portait sur les quatre thèmes suivants auxquels, chaque fois, se référaient trois questions semi-ouvertes :

- les principales caractéristiques de leurs attitudes managériales actuelles,

- les bonnes pratiques de management,

- les pré-requis du bon manager,

- les bonnes attitudes professionnelles des managers.

L'analyse des réponses fermées aux douze questions ont été analysées à partir d'un outil statistique (SPSS Statistics Version 20.0) afin de réaliser à la fois une étude descriptive et factorielle des réponses obtenues. A partir de l'administration d'un questionnaire visant à étudier l'importance relative d'un ensemble structuré de variables (Conway et Huffcutt, 2003), Pan et al (2012) préconise l'utilisation d'un analyse en composante principale. Nous avons repris cette approche en lui associant une régression multiple uniquement pour mettre en évidence l'importance relative de chaque variable identifiée par notre analyse en composante principale.

Sur le plan méthodologique, dans le cas de notre étude, nous suggérons qu'une analyse quantitative d'une enquête portant sur l'identification de facteurs culturels peut être favorablement complétées par une analyse qualitative des questions ouvertes ou des précisions aux questions fermées formulées par les managers. Pour cela, nous proposons, dans un deuxième temps, d'analyser les réponses ouvertes en recherchant dans les verbatim encodés grâce au logiciel n'Vivo l'existence de relations entre les facteurs identifiés par notre analyse quantitative et les principaux rites contenus dans les écrits culturels et religieux chinois. Cette analyse qualitative complémentaire vise à répondre aux exigences de notre cadre conceptuel (Collins, 2004) en repérant les relations entre le contenu des rites et leurs effets sur les comportements des managers.

Le recours à cette double analyse quantitative et qualitative vise à répondre aux difficultés inhérentes à l'analyse des comportements managériaux en Chine mis en avant par Adler et al. (1989). Ces auteurs réfutent la possibilité d'interprétation des analyses en composante principale menées en Chine par des chercheurs occidentaux en considérant que la structure des réponses des managers chinois peut être bimodale et non normale c'est à dire diamétralement opposée sans que l'analyse factorielle puisse en rendre compte. Pour limiter ce biais interprétatif, nous avons, d'une part, mobilisé les travaux antérieurs menés sur les valeurs culturelles chinoises (Pan et al., 2012) pour « siniser » nos hypothèses initiales et, d'autre part, nous avons utilisé les connaissances des cultures occidentales et chinoises des deux auteurs 
par l'adjonction d'une étude qualitative des verbatim obtenus en vue d'infirmer ou confirmer les résultats de notre ACP (Analyse en Composante Principale) et de notre régression multiple.

\section{Résultats : Une analyse culturelle des comportements managériaux en Chine}

Notre cadre méthodologique a été conçu à partir des réponses des managers de la maison mère $(40 \% \mathrm{du}$ total des cadres et ingénieurs du groupe) et de ses six filiales chinoises dont deux à capitaux mixtes dont les cadres représentent 20,26\% de notre échantillon (31 cadres répondants sur 153). Le choix de cet échantillon se justifie par, à la fois, la persistance de l'intervention publique dans l'économie entrepreneuriale, mais, aussi, par l'ouverture du capital des entreprises d'Etat et collectives surtout à partir $\mathrm{XVI}^{\mathrm{e}}$ Congrès du Parti communiste chinois en novembre 2002 faisant suite à l'entrée de la Chine dans l'OMC. Une analyse même introductive des comportements des managers chinois imposant sans doute d'interroger les proximités ou les distances managériales selon les formes de détention de capital des entreprises.

L'analyse sociologique des répondants peuvent être synthétisée de la manière suivante :

$\underline{\text { Tableau } 1}$ : caractéristiques sociologiques des répondants $(\mathrm{n}=153)$

\begin{tabular}{|c|c|c|c|c|c|}
\hline Age des managers & Effectifs & $\%$ & Profession & Effectifs & $\%$ \\
\hline moins de 30 ans & 24 & 15.68 & Responsable de service & 12 & 7.84 \\
\hline entre 30 et 40 ans & 56 & 36,60 & Cadre financier et adm & 14 & 9.15 \\
\hline entre 40 et 50 ans & 49 & 32,03 & Cadre de Production & 31 & 20.26 \\
\hline entre 50 et 60 ans & 17 & 11,11 & Cadre commercial & 52 & 33.99 \\
\hline Plus de 60 ans & 7 & 4.58 & Cadre RH & 20 & 13.07 \\
\hline \multirow[t]{2}{*}{ Total } & 153 & 100 & Autres cadres & 24 & 15.69 \\
\hline & & & Total & 153 & 100 \\
\hline Pratiques religieuses & Effectifs & $\%$ & & & \\
\hline Bouddhisme & 17 & 11,11 & & & \\
\hline Confucianisme & 17 & 11,11 & & & \\
\hline Taoïsme & 36 & 23,53 & & & \\
\hline Non pratiquant & 83 & 54,25 & & & \\
\hline Total & 153 & 100 & & & \\
\hline
\end{tabular}

Notre échantillon porte sur une part conséquente de cadre administratifs et commerciaux $(70 \%$ des répondants). Ceci est assez caractéristique de l'évolution du management en Chine depuis 15 ans notamment dans les villes émergentes (villes de l'ouest et sud-ouest du pays) pour lequel les cadres et 
ingénieurs de production installés par le pouvoir communiste dans les années 70-80 ont été remplacés, avec l'ouverture progressive du capital des entreprises publiques, par des cadres administratifs formés dans les universités du pays et étrangères (Japon et USA notamment). L'existence de seulement 7 cadres formés dans des universités étrangères sur l'ensemble de notre échantillon (153 cadres) ne nous permet pas de mener une analyse spécifique de leurs réponses. Par contre, une analyse de leurs seules réponses a pu révéler une conformité de leurs réponses avec celles des autres cadres de l'échantillon.

Une analyse plus approfondie de cet échantillon fait apparaitre que la plupart des managers de ce groupe sont majoritairement âgés de 30 à 50 ans (78\% des répondants) et ils reconnaissent ne pas être pratiquant religieux (à 54,25\%).

L'importance des rites culturels est particulièrement confirmée par l'analyse factorielle en composante principale que nous avons opérée et qui met en évidence six facteurs explicatifs des comportements des managers dans ces entreprises chinoises.

Ainsi, les principaux facteurs décrivant les comportements des managers chinois de ces organisations que l'on peut retenir à partir de l'analyse factorielle et du seuil de signification de Cronbach sont les suivants :

- N1 le respect du guanxi

- N2 La qualité des relations humaines

- N3 Le respect de la hiérarchie

- N4 L'harmonie collective

- N5 Le respect de la « face»

- N6 Le respect des anciens ou des ancêtres

En appliquant une régression linéaire sur les six composants ci-dessus il est possible de mettre en évidence les rites culturels qui peuvent expliquer les comportements managériaux. Par la suite, ces éléments pourront être retranscrits sous la forme d'une équation afin de mettre en évidence l'importance relative accordée dans ces structures à chacun des six facteurs identifiés :

$$
\mathrm{Y}=\mathrm{B} 0+\mathrm{B} 1 \mathrm{N1}+\mathrm{B} 2 \mathrm{N2}+\mathrm{B3N3}+\mathrm{B} 4 \mathrm{~N} 4+\mathrm{B} 5 \mathrm{~N} 5+\mathrm{B} 6 \mathrm{~N} 6
$$

Les six facteurs explicatifs sont représentés par les variables N1 à N6; B1 à B6 représentent les coefficients de signification propres à chacun des facteurs, B0 représente la constante d'évolution pour l'ensemble des facteurs. Le coefficient R-carré (ajusté) de la régression linéaire multiple est égal à 0.67 , cela signifie que les six composants retenus expliquent $67 \%$ des comportements managériaux de notre échantillon, ce qui permet de confirmer la fiabilité du modèle proposé. Ce dernier ne retenant que les variables indépendantes (tableau 2) présentant un niveau satisfaisant de signification (test de Student supérieur à 2) et une faible colinéarité (seuil de tolérance $>0.3$ et $\mathrm{VIF}<3$ ).

Le tableau suivant donne les résultats de la régression linéaire déterminant le coefficient Beta de chacun des facteurs qui permettent de mesurer l'importance relative de chacun des facteurs dans les 
comportements des managers. Les six facteurs identifiés de N1 à N6) présentent tous un alpha de Cronbach et un indice KMO (Kaiser-Meyer-Olkin) supérieurs à 0,60 (tableau 2) nécessaires à la justification d'une corrélation forte entre les variables des facteurs et la variable dépendante "y» représentant les comportements managériaux dans les entreprises étudiées.

Tableau 2 : Résultats de l'analyse de régression

\begin{tabular}{|c|c|c|c|c|c|}
\hline \multicolumn{3}{|c|}{ Coefficients non standardisés } & Coefficients & \multicolumn{2}{|c|}{ Statistiques de colinéarité } \\
\hline \multicolumn{2}{|l|}{ A } & Erreur & Bêta & Seuil de tolérance & VIF \\
\hline (Constante) y & $-5,795 \mathrm{E}-$ &, 000 & & & \\
\hline N1 &, 125 & 000 & ,203 & ,617 & 1,555 \\
\hline $\mathrm{N} 2$ &, 125 &, 000 &, 186 & ,802 & 1,120 \\
\hline N3 &, 125 &, 000 &, 267 & ,665 & 2,391 \\
\hline $\mathrm{N} 4$ &, 125 &, 000 &, 181 & ,713 & 1,349 \\
\hline N5 &, 125 &, 000 &, 199 &, 824 & 2,100 \\
\hline N6 &, 125 &, 000 & ,224 & 670 & 1,875 \\
\hline \multicolumn{3}{|c|}{ Cronbach's Alpha } & 0,961 & & \\
\hline \multicolumn{3}{|c|}{ KMO (Kaiser-Meyer-Olkin) } & 0.821 & & \\
\hline
\end{tabular}

Ceci nous permet de présenter une équation explicative des comportements managériaux dans ce groupe qui prend la forme suivante :

$$
\mathrm{Y}=-\mathbf{5 , 7 9 5} \mathrm{E}^{\mathrm{E}}-16+0,203 \mathrm{~N} 1+0,186 \mathrm{~N} 2+0,267 \mathrm{~N} 3+0,181 \mathrm{~N} 4+0,199 \mathrm{~N} 5+0,224 \mathrm{~N} 6
$$

L'équation illustre l'importance accordée par les managers principalement à l'harmonie collective (N3), puis au respect des anciens et des ancêtres (N6) et au respect du guanxi et de la «face » (N1 et N5). L'analyse que nous proposons ici décrit une importance porosité entre ces déterminants qui se retrouvent à la fois dans les écrits classiques et les ouvrages religieux en accordant une place essentielle aux rites culturels qui les fédèrent alors même que, dans la littérature, ce rôle est uniquement attribué au confucianisme voire au taoïsme.

Ceci est confirmé par notre analyse qualitative que nous avons menée à partir de l'encodage N'Vivo des principales réponses ouvertes. Le tableau 3 synthétise cette analyse en mettant en évidence les conséquences managériales de l'observance des rites culturels par les managers du groupe que nous avons étudié. Après leur encodage, Les verbatim recueillis ont pu être rattachés à un certain nombre d'écrits culturels et religieux chinois qui déterminent en partie les comportements managériaux. 
Tableau 3 : Analyse de l'influence des rites culturels d'interaction sur le management en Chine

\begin{tabular}{|c|c|c|}
\hline Verbatim & $\begin{array}{l}\text { Valeurs culturelles } \\
\text { correspondantes }\end{array}$ & $\begin{array}{c}\text { Conséquences } \\
\text { managériales }\end{array}$ \\
\hline $\begin{array}{l}\text { "Le chef est considéré comme un parent» } \\
\text { "l'organisation de l'entreprise est calquée sur } \\
\text { celle de la famille» }\end{array}$ & $\begin{array}{l}\text { Respect du guanxi et des } \\
\text { ancêtres (confucianisme) }\end{array}$ & $\begin{array}{l}\text { Ritualiser les rôles de } \\
\text { chaque membre de } \\
\text { l'organisation }\end{array}$ \\
\hline $\begin{array}{l}\text { "Le manager est celui qui, par la respectabilité de } \\
\text { sa pensée, doit rechercher l'équilibre des décisions } \\
\text { " } \\
\text { «tre juste et équilibré » }\end{array}$ & $\begin{array}{l}\text { Harmonie sociale } \\
\text { (Confucianisme) }\end{array}$ & $\begin{array}{l}\text { Privilégier l'informel et la } \\
\text { souplesse des relations de } \\
\text { proximité }\end{array}$ \\
\hline $\begin{array}{l}\text { "Être à la fois intérieur et extérieur" } \\
\text { " être yin et yang dans son travail mais aussi dans } \\
\text { sa vie" }\end{array}$ & $\begin{array}{l}\text { Yin et Yang (taoïsme) } \\
\text { Équilibre des contraires }\end{array}$ & $\begin{array}{l}\text { Rechercher la } \\
\text { complémentarité entre } \\
\text { espace public et espace } \\
\text { privé }\end{array}$ \\
\hline $\begin{array}{l}\text { "La culture chinoise impose des espaces de } \\
\text { négociation " } \\
\text { "nous devons être à l'écoute des employés » }\end{array}$ & $\begin{array}{l}\text { Culte de la nature et du non } \\
\text { agir (Livre des mutations, } Y i \\
\text { Jing) }\end{array}$ & $\begin{array}{l}\text { Laisser des espaces de } \\
\text { liberté face aux règles } \\
\text { officielles }\end{array}$ \\
\hline $\begin{array}{l}\text { «Donner l'exemple » } \\
\text { "Contrôler sa parole » } \\
\text { « Rapprocher parole et raison » }\end{array}$ & $\begin{array}{l}\text { Orientation collective } \\
\text { (confucianisme) } \\
\text { (Livre des documents, } S h u \\
\text { Jing) }\end{array}$ & $\begin{array}{l}\text { Rapprochement des } \\
\text { comportements } \\
\text { privés/comportements } \\
\text { publics }\end{array}$ \\
\hline $\begin{array}{l}\text { «Suivre sa voie dans ses attitudes de manager» } \\
\text { «Appliquer ses propres valeurs dans son métier»" }\end{array}$ & La voie (Taoïsme) & $\begin{array}{l}\text { Obtenir la réflexivité de } \\
\text { l'action à partir de valeurs } \\
\text { privées }\end{array}$ \\
\hline $\begin{array}{l}\text { " vérifier que les salariés détiennent les } \\
\text { compétences d'un bon chinois » }\end{array}$ & $\begin{array}{l}\text { La ritualisation des valeurs } \\
\text { Livre des rites (Liji) }\end{array}$ & $\begin{array}{l}\text { Ritualiser les } \\
\text { comportements moraux }\end{array}$ \\
\hline
\end{tabular}

\section{Discussions}

Les quatre variables mises en évidences par notre enquête (guanxi, face, Harmonie collective et respect des ancêtres) sont représentatives des caractéristiques des rites d'interaction définies par Collins (2004) dans son modèle des rites d'interaction. Nous avons en effet constaté, à travers notre enquête, que ces éléments de la culture chinoise, de manière transverse, influencés les comportements des managers de manière prépondérante et qu'ils puisaient leurs origines dans la tradition culturelle chinoise (Livres classiques notamment) tout en étant ensuite compilés dans les principaux écrits religieux.

Ces quatre variables en symbolisant chacune des caractéristiques des rites d'interaction mises en avant par Collins (appartenance à un groupe, barrières à l'entrée, centre d'attentions, humeurs communes ; figure 2), permettent de construire les conséquences (outcomes) des rites d'interaction identifiés par cet auteur 
(figure 2).

Cette identification des complémentarités entre chacun des rites permet de postuler l'existence de rites d'interaction (au sens de Goffman et Collins) présentant une certaine homogénéité et des convergences communes. Ceci permet, grâce à notre analyse des réponses ouvertes à notre enquête (tableau 3), de débuter l'identification de rituels managériaux représentatifs des comportements des managers que nous avons interrogés (figure 2).

Notre méthodologie à consister à rattacher aux quatre principaux rites d'interaction mis en évidence par notre analyse en composante principale (harmonie collective, guanxi, face et respect des ancêtres) les rituels managériaux issus de notre analyse des verbatim et des valeurs culturelles correspondantes (tableau $3)$.

\section{Figure 2 : Les rituels managériaux issus de la culture traditionnelle chinoise}

\begin{tabular}{|c|c|c|}
\hline $\begin{array}{c}\text { Caractéritiques des rites } \\
\text { d'interaction }\end{array}$ & $\begin{array}{l}\text { Conséquences des rites } \\
\text { d'interaction }\end{array}$ & Rituels managériaux \\
\hline $\begin{array}{l}\text { Appartenance à un groupe } \\
\text { (l'harmonie collective) }\end{array}$ & Solidarité de groupe & $\begin{array}{l}\text { - Définir la place et le rang de chaque } \\
\text { membre de l'organisation } \\
\text { - Rapprocher les espaces privé et } \\
\text { public }\end{array}$ \\
\hline $\begin{array}{l}\text { Barrières à l'entrée } \\
\quad \text { (guanxi) }\end{array}$ & Transmission d'énergie emotionnelle & $\begin{array}{l}\text { Favoriser la réflexivité dans l'action } \\
\text { plus que le respect des procédures }\end{array}$ \\
\hline $\begin{array}{l}\text { Centres d'attention communs } \\
\text { (la Face) }\end{array}$ & $\begin{array}{l}\text { Symboles communs de relations } \\
\text { sociales }\end{array}$ & $\begin{array}{l}\text { Souplesse de relations intra } \\
\text { organisationnelles et réactivité de } \\
\text { l'organisation }\end{array}$ \\
\hline $\begin{array}{l}\text { Humeurs communes } \\
\text { (le respect des ancêtres) }\end{array}$ & Règles de moralité & $\begin{array}{l}\text { Priorité de la moralité des individus } \\
\text { sur la règle écrite }\end{array}$ \\
\hline
\end{tabular}

Dans les lignes qui suivent, nous proposons de revenir sur chacune de ces quatre variables représentatives des principaux rites culturels d'interaction sociale respectés par les managers interrogés afin d'expliquer en quoi elles déterminent les rituels managériaux que nous identifions par la suite.

\subsection{L'importance des rites dans les relations interpersonnelles (guanxi)}

Le confucianisme est largement utilisé pour expliquer la force de la famille et du lien social dans tous types de relations interpersonnelles dans un rapport de respect en fonction du titre ou de l'âge de la personne (Lévi, 2003 ; Cheng 1997, p. 38). Force est de constater que le rapport à la famille et au guanxi revient de manière récurrente dans les études soit pour comprendre la société chinoise (Padovani, 2000), expliciter un mode de management de type confucéen (Levi, 2011), ou rendre compte des techniques efficientes de management interculturel (Piques, 2001; Faure, 2004).

Le résultat de nos enquêtes et l'analyse des verbatim semblent décrire des situations professionnelles où 
l'insertion dans un réseau est prépondérante mais elle n'est pas nécessairement définie à partir de valeurs confucéennes d'ordre et de hiérarchie. S'il y a respect d'une hiérarchie c'est souvent plus en référence au respect des rôles au sein du guanxi très signifiant en Chine et qui explique un grand nombre de comportements comme celui de s'effacer en fonction de la position sociale de ceux-ci. Seul le respect de ces rôles et de la hiérarchie familiale peuvent engendrer l'harmonie et l'ordre. Les rites et la nécessité de leur respect ne peuvent être compris qu'en fonction de ce principe de régulation sociale (Barbier, 2011). L'action ou l'inaction en entreprise, si elles se réalisent à partir à de considérations de rang, se déterminent plus à partir de la hiérarchie familiale dans laquelle s'inscrivent potentiellement les supérieurs hiérarchiques ou certains collègues issus de la même « branche » familiale.

Les verbatim relatif à la personnalité du chef, présenté comme un membre de la famille, ou de l'organisation de l'entreprise proche de celle de la famille (tableau 3 ; figure 2) soutiennent l'influence dans le management en Chine des rites culturels du guanxi et du respect des ancêtres.

\subsection{Les rites de respect des ancêtres précèdent l'ordre et la hiérarchie}

L'ordre ou la hiérarchie sont loin d'être centraux dans la prise de décision et l'action entrepreneuriale. Au contraire, l'appel au réseau familial renvoie plutôt à la recherche d'une action équilibrée en recherchant dans la ritualisation du respect des ancêtres une définition des positions hiérarchiques de chaque membre de l'organisation (tableau 3 ; figure 2).

Dans la société chinoise, s'il y a hiérarchie, c'est plus un ordre transitoire en quête d'un équilibre futur entre des valeurs et des références multiples telles que le préconisent les valeurs culturelles reprises par les managers que nous avons interrogés (tableau 3).

Le respect d'un certain ordre dans les entreprises s'explique par la place que les éléments de la nature et les ancêtres imposent de modestie et d'humilité aux individus. C'est par un retour régulier vers la puissance de la nature (ciel et terre) et de la parole des ancêtres que l'individu va construire sa propre réflexivité dans son activité professionnelle.

Il faudrait vérifier si le déterminisme de la hiérarchie dans les comportements des managers chinois ne pourrait pas correspondre plus précisément à des emprunts managériaux à d'autres modèles asiatiques de gestion notamment japonais. En effet, dans les années 80, un grand nombre de managers actuels ont été envoyés au Japon par le gouvernement chinois pour clôturer leurs études et s'imprégner du modèle de management japonais inspiré lui aussi par le culte des ancêtres mais également par des méthodes d'organisation spécifiques. Il nous semble qu'un grand nombre de comportements ostentatoires de type militaire comme l'interprétation par les salariés d'un hymne d'entreprise en début de journée proviennent simplement d'une reproduction ou d'une sinisation de l'organisation hiérarchique de l'entreprise japonaise. 
L'objectif de cet affichage est de faciliter en interne une gestion plus désordonnée des actions professionnelles que l'on sait inévitablement aléatoire car elle incarne le changement naturel propre à toute activité.

Le « livre des mutations » (Yi Jing, -1027 av JC, tableau 3) illustre parfaitement cette idée de changement perpétuel imposé par la nature et qui ne peut s'équilibrer que de manière complexe à partir de valeurs communes que les croyances religieuses (confucianisme, bouddhisme, taoïsme) ne peuvent pas exprimer à elles seules. Plus assurément, ces valeurs nous renvoient sans doute à une histoire plus ancienne, celle des traditions culturelles chinoises décrites dans les premiers ouvrages apparus en Chine à partir du XVème siècle av JC (livre de l'histoire, livre des mutations, livre des saisons) bien avant les écrits de Confucius et de Lao Tseu (Vème siècle av JC).

\subsection{Le respect de la "face" : forme ritualisée du respect d'autrui}

Pour illustrer encore cette importance des rites d'interaction chinois dans les actions personnelles ou professionnelles, nous pouvons également approcher la «face» décrite par de nombreux auteurs en management comme un élément fondamental du comportement professionnel des chinois. La «face» chinoise est souvent définie comme l'élément fondateur des interactions sociales chinoises.

Les chinois perdent la "face» lorsqu'ils dérogent aux règles, aux rites, aux us et coutumes de leur communauté et des relations qui la constituent (guanxi). En référence au rite du respect de la "face », les chinois, dans leur champ extraprofessionnel comme professionnel, se perçoivent comme un champ de relations et d'interactions (Goffman, 1974, p.13).

Il faut intégrer dans l'analyse que l'on fait des comportements managériaux que la vie d'un individu sert essentiellement à préparer sa vie future d'esprit après sa mort (Jullien, 2012). Perdre la «face » en famille signifie perdre une occasion d'être honoré comme un ancêtre important, c'est une menace de mise au ban de la famille, et représente une gravité extrême au regard de la longueur de sa vie future d'esprit isolé.

Ce rite d'interaction par le respect de la «face » prône au contraire une certaine retenue et une humilité afin de faciliter l'effacement de l'individu par rapport à la puissance de la famille et de la nature. La « face » chinoise en entreprise est donc plus à rapprocher du «masque » utilisé dans les rites ancestraux ou tribaux et destiné à se protéger d'un engagement individuel trop important. Perdre la "face » ne consisterait pas nécessairement à perdre sa dignité ou son prestige mais plutôt à faire preuve d'une immodestie ou d'un manque de discrétion ne permettant pas de faire place aux autres membres du réseau familial des vivants ou non vivants. La «face» peut être apparentée à un comportement favorable à la concertation plus qu'un frein ou une limite à la négociation.

\subsection{L'harmonie collective : un comportement ritualisé en entreprise}


La pérennité apparaît être une valeur référence de la pensée chinoise et elle s'obtient dans l'esprit des salariés et managers chinois par un retour cyclique aux rites d'harmonie et d'orientation collective représentant «la culture chinoise qui impose des espaces de négociation » (tableau 3). Dans l'entreprise, cela a une conséquence importante : le respect de la hiérarchie est une pratique rituelle qui donne aux rites force de normes plutôt que l'obéissance aveugle à un supérieur au nom d'un ordre prescrit notamment par le recherche de l'harmonie collective. En matière de management, plutôt que de chercher à opposer les rites, les chinois se préoccuperont de les rendre harmonieux au nom d'une complémentarité entre espace public et privé (tableau 3 ; figure 2).

Pour les salariés et managers chinois, un système de rite est toujours profitable alors qu' «aucune croyance n'a d'intérêt véritable : les différents systèmes de croyance importent peu » (Granet, 1922, p. 116).

\section{Conclusion}

Nous avons souhaité mettre en avant dans cet article la place signifiante dans le management en Chine du respect de certaines valeurs culturelles historiquement et traditionnellement définis par le concept de rite (li). En mobilisant, le modèle des rites d'interaction (Collins, 2004), nous avons tenté de repérer les connexions existantes entre le contenu de ces rites culturels d'interaction et leur ritualisation dans le cadre des pratiques managériales en Chine.

En termes managériaux, cette description de l'influence des rites d'interaction ouvre des perspectives d'objectivation de phénomènes de ritualisation du management en Chine pour essayer de répondre à un certain nombre d'interrogations soulevé, dès à présent, par le management interculturel en Chine ou hors de ses frontières.

Par ailleurs, les entreprises chinoises offrent la particularité de mettre en évidence certaines régularités comportementales des managers qui, sur le plan théorique, autorisent une description plus précise de la manière dont les principes et rituels managériaux se construisent culturellement.

En s'inscrivant au centre des déterminants culturels des chinois, les rites culturels d'interaction, par leurs appariements, déterminent un ensemble de valeurs culturelles qui dépassent le cadre des religions officielles sans pour autant les nier. Ceci permet de proposer une vision élargie du lien entre management et culture en Chine.

Les difficultés d'analyse des facteurs culturels influençant le management chinois semblent encore aujourd'hui largement irrésolues (Adler et al, 1989). Face à la complexité et l'étendue de ces difficultés, nous avons essayé de tester des précautions méthodologiques complémentaires en s'appuyant sur les résultats de travaux antérieurs récents (Pan et al., 2012) et en associant une analyse quantitative et 
qualitative à partir des compétences spécifiques des deux auteurs de cet article. Nos résultats semblent justifier les potentialités de l'association de cette posture méthodologique avec le cadre conceptuel des rites d'interaction proposé par Collins (2004) pour débuter l'identification de rituels managériaux en Chine. Par contre, il subsiste certains doutes sur la capacité de cette association à produire des résultats sur des échantillons de managers plus larges ou présentant un niveau d’hétérogénéité plus étendu.

Face à l'importance des biais méthodologiques observables dans ce type de travaux, nous considérons que leur résolution à long terme peut provenir de contributions limitées et partielles telles que celle que nous proposons ici à la double condition de les rapprocher fortement des résultats des études antérieures et de les positionner, dans l'avenir, en tant que programme de recherche à part entière.

\section{Références}

Adler N. J., Campbell N., et Laurent A. (1989), In search of appropriate methodology: From outside the People's Republic of China looking in, Journal of International Business Studies, p. 61-74.

Ams B. (2008), Les nouvelles pratiques du business en Chine, Paris, Maxima.

Ascencio C. et Rey D. (2010), Etre efficace en Chine : Le management à l'épreuve de la culture chinoise, Paris, Pearson Education France.

Barbier R. (2001), Chine, environnement et philosophie, in Ecoformation, éducation permanente sur le web (http://www.barbier-rd.nom.fr/articlechne5.rtf.pdf)

Barbier R. (2011), Le management émancipant, une philosophie interculturelle du sens du travail humain, (de l'imaginaire leurrant à l'utopie créatrice).in F. Fourcade et M. Krichewsky, Former les managers,Pratiques de Formation/Analyse, , $\mathrm{n}^{\circ}$ 60- 61 (juillet - décembre), Université Paris 8, p. 183-230 Barney J. B. et Zhang, S. (2009), The Future of Chinese Management Research: A Theory of Chinese Management versus A Chinese Theory of Management, Management and Organization Review, vol. 5, $\mathrm{n}^{\circ}$ 1, p. 15-28. doi: 10.1111/j.1740-8784.2008.00102.x

Bartuneck J.M. (1984), Changing interpretative schemas and organizational restructuring: The example of a religious order, Administrative Science Quarterly, n²9, p. 355-372.

Benveniste E. (1969), Le vocabulaire des institutions indo-européennes, Paris, Ed. de Minuit,, t. 2, pp. 267-272.

Billeter J. F. (2006), Contre François Jullien, Paris, Éditions Allia.

Bruner J., Chen J., Sun C., Zhou N. (1989), The role of Guanxi in negotiations in the Pacific Bassin, Journal of Global Marketing, vol. 3, $\mathrm{n}^{\circ} 2$.

Cazal D., Davoine, E., Louart P., Chevalier F. (2010), GRH et mondialisation: nouveaux contextes, nouveaux enjeux, Collection AGRH, Paris, Vuibert.

Cheng A. (1997), Histoire de la pensée chinoise, Seuil, Paris.

Cheng A. (dir) (2007), La pensée en Chine aujourd'hui, Paris, Gallimard, folio essais.

Collins, R. 2004. Interaction Ritual Chains. Princeton University Press

Conway J. M. et Huffcutt, A. I. (2003), A review and evaluation of exploratory factor analysispractices in organizational research, Organizational Research Methods, vol. 6, n², p. 147-168.

Cuche D. (2004), La notion de culture dans les sciences sociales (2 éd.), Paris, La Découverte.

d'Iribarne, P (2011), les cultures et les performances, Réalités industrielles, Annales des Mines, vol. 2, p. $18-23$

Drucker P. (1954), La pratique de la direction des entreprises, Paris, Éditions d'Organisation.

Drucker P. (1993), Au-delà du capitalisme, Paris, Dunod.

Dumézil G. (1966), La religion romaine archaïque, Paris, Payot. 
Durkheim E. (1894), Les Règles de la méthode sociologique, Paris, Payot, coll. "Petite Bibliothèque Payot".

Fan Y. (2000), A classification of Chinese culture, International Journal of Cross Culture Management, vol. $7, n^{\circ} 2$, p. 3-10.

Fang T. (2012), Yin Yang: A new perspective on culture, Management and Organization Review, vol.8, n'1, p. $25-50$.

Faure G.O. et Chen D. (1997), Chinese Negotiators: profiles and behaviors, Journal of Euro-Asian Management, vol. $3, \mathrm{n}^{\circ} 2$.

Faure G.O. (2004), Approcher la dimension interculturelle en négociation internationale. Revue française de gestion, (6), 187-199.

Faure S. (2003), Manager à l'école de Confucius, Paris, Editions d'Organisations.

Fernandez B. et Zheng L. (2008), Culture et Gestion en Chine, in Davel, E., Culture et gestion dans le monde contemporain : la plongée interculturelle, Pul-Teluq, Québec.

Fustel de Coulanges N.-D. (1864), La Cité antique : Etude sur le culte, le droit, les institutions de la Grèce et de Rome, publié avec le concours du Centre national des lettres, Paris, Flammarion, 1984.

Gernet J. (2001), La Chine ancienne, « Que sais-je ? », édition PUF, Paris.

Goffman E. (1974), Les rites d'interaction, Paris, Les Editions de Minuit.

Goxe F. (2012). Innovation with 'Chinese'characteristics ? Reflecting on the implications of an ethnicbased paradigm of management and innovation, Prometheus, vol.30, $n^{\circ} 2$, p. 155-168.

Goossaert, V. (2007), L'invention des «religions» en Chine moderne, In A. Cheng (dir), La pensée en Chine aujourd'hui, p. 185-213, Paris, Gallimard, folio essais.

Granet M. (1922), La Religion des Chinois, Paris, PUF.

Granet M. (1968), La pensée chinoise, Paris, Editions Albin Michel.

Héran F. (1986), Le rite et la croyance, Revue française de sociologie, vol. 27, n², p. 231-26

Hwang K. K. (2006), Moral face and social face: Contingent self-esteem in Confucian society.

International Journal of Psychology, vol. 41, $n^{\circ} 4,276-281$.

Hwang, K. K. (2011), Foundations of Chinese psychology: Confucian social relations, vol. 1, Springer.

Jullien F. (2012),Cinq concepts proposés à la psychanalyse, Hachette. com.

Knutsson J. (1986), Chinese commercial negotiating behaviour and its institutional and cultural determinants,in Chinese Culture and Management, ECAM, Euro-China Association for Management Development, Bruxelles.

Kulich S.J. et Zhang R. (2010), The multiple frames of 'Chinese' values: From tradition to modernity and beyond. In M. H. Bond (Ed.), The Oxford handbook of Chinese psychology, New York, Oxford University Press.

Lavin F. (1994), Negotiating with the chinese, Foreign affairs, vol. 73, $\mathrm{n}^{\circ} 4$.

Lévi J. (2003), Confucius, Albin Michel, Paris.

Levi, J. (2011), Confucius vu par ses détracteurs. Religions \& Histoire, n³8, p.32-37.

Lilla M. (2010), The Stillborn God, Religion, Politics and the Modern West, New York, Knopf, traduction française : Le Dieu mort-né, Paris, Seuil.

Lin Y. (1997), La chine et les chinois, Paris, Payot.

Mauss M. (1947), Manuel d'ethnographie, présenté par D. Paulme, Paris, rééd. Payot, 1967.

Mauss M. (1950), Sociologie et anthropologie, introd. de C. Lévi-Strauss, Paris, PUF, rééd.1973.

Padovani F. (2000), Les guanxi au cœur de la société chinoise. pp.78-84 in Chine-France : approche interculturelles en économie, littérature, pédagogie, philosophie et sciences humaines, (Coll.) Zheng L.H. et Desjeux D., Paris, L'Harmattan.

Pan Y., Rowney J. A. et Peterson M. F. (2012), The structure of Chinese cultural traditions: An empirical study of business employees in China, Management and Organization Review, vol. 8, $n^{\circ} 1$, p. 77-95.

Pareto V. (1917), Traité de sociologie générale, Genève, librairie droz, réédition 1968. 
Pesqueux, Y. (2010), Ambiguïté de la culture, ambiguïté de la rationalité. La rationalité-Mythes et réalités, p.149-175.

Pesqueux, Y., Martinet, A-C. (2013), Epistémologie des sciences de gestion, Paris, Vuibert.

Piques M. C. (2001), Les miroirs de la négociation en Chine : voyage dans l'univers mental et social chinois, Arles, Éditions Philippe Picquier.

Sainsaulieu, R (1988), L'identité au travail, Paris, FNSP.

Scheid, J. (1985), Religion et piété à Rome, Paris, La Découverte.

Thietart RA. (2007), Méthodes de recherche en Management, Paris, Dunod.

Wang J. H. (1981), Some Cultural Factors Affecting Chinese in Treaty Negotiation, in Anand (ed), Cultural Factors in International Relations, New Delhi.

Weber M. (1917), Essai sur quelques catégories de la sociologie comprehensive, in Essais sur la théorie de la science, Paris Plon, 1965.

Weber M. (1921), Economie et société, Paris, Plon, 2, édition 1971.

Xing F. (1995), The Chinese cultural system: Implications for cross-cultural management,SAMAdvanced Management Journal,vol. 60, n¹, p. 14-20.

Yang L.S. (1957), The concept of Pao as basis for social relations in China, in Fairbank J.K. (ed), Chinese thought and institution, Chicago, Chicago University Press.

Yau O. H. M. (1994), Consumer behavior in China: Customer satisfaction and culturalvalues, London and New York, Routledge

Yeh K. H. et Bedford O. (2004), Filial Belief and Parent-Child Conflict, International J. of Psychology, vol. 39, p. 132-144 


\section{ANNEXE \\ ENQUETE AUPRES DES MANAGERS}

I- les principales caractéristiques de leurs attitudes managériales actuelles

Question 1 : Quelles sont vos principales motivations à exercer votre métier ?

(veuillez entourer le chiffre correspondant à votre choix)

\begin{tabular}{|l|l|}
\hline gagner plus d'argent & pas du tout d'accord 12345 tout à fait d'accord \\
\hline $\begin{array}{l}\text { avoir une nouvelle } \\
\text { expérience professionnelle }\end{array}$ & pas du tout d'accord 12345 tout à fait d'accord \\
\hline $\begin{array}{l}\text { améliorer votre position } \\
\text { dans la hiérarchie familiale }\end{array}$ & pas du tout d'accord 12345 tout à fait d'accord \\
\hline Ne pas perdre la face & pas du tout d'accord 12345 tout à fait d'accord \\
\hline $\begin{array}{l}\text { apprendre de nouvelles } \\
\text { choses }\end{array}$ & pas du tout d'accord 12345 tout à fait d'accord \\
\hline $\begin{array}{l}\text { Vous faire de nouvelles } \\
\text { relations }\end{array}$ & pas du tout d'accord 12345 tout à fait d'accord \\
\hline Autres (précisez)
\end{tabular}

Question 2 : Confronté à des choix ou ou une prise des décisions, j'essaie avant tout de (veuillez entourer le chiffre correspondant à votre choix)

\begin{tabular}{|l|l|}
\hline $\begin{array}{l}\text { Prendre une décision } \\
\text { harmonieuse }\end{array}$ & pas du tout d'accord 12345 tout à fait d'accord \\
\hline respecter la hiérarchie & pas du tout d'accord 12345 tout à fait d'accord \\
\hline Ne pas perdre la face & pas du tout d'accord 12345 tout à fait d'accord \\
\hline etre créatif & pas du tout d'accord 12345 tout à fait d'accord \\
\hline $\begin{array}{l}\text { de respecter mes ancêtres et leurs } \\
\text { images }\end{array}$ & pas du tout d'accord 12345 tout à fait d'accord \\
\hline Je respecte les procédures & pas du tout d'accord 12345 tout à fait d'accord \\
\hline De faire appel à mon expérience & pas du tout d'accord 12345 tout à fait d'accord \\
\hline Autres (précisez) & \\
\hline
\end{tabular}

Question 3 : A travers les relations avec vos salariés, vous recherchez principalement (veuillez entourer le chiffre correspondant à votre choix)

\begin{tabular}{|l|l|}
\hline A être respecté & $\begin{array}{l}\text { pas du tout d'accord } 12345 \text { tout à fait } \\
\text { d'accord }\end{array}$ \\
\hline À vous faire des amis & $\begin{array}{l}\text { pas du tout d'accord } 12345 \text { tout à fait } \\
\text { d'accord }\end{array}$ \\
\hline A obtenir une reconnaissance sociale & $\begin{array}{l}\text { pas du tout d'accord } 12345 \text { tout à fait } \\
\text { d'accord }\end{array}$ \\
\hline A ne pas perdre la face & $\begin{array}{l}\text { pas du tout d'accord } 12345 \text { tout à fait } \\
\text { d'accord }\end{array}$ \\
\hline A vous épanouir personnellement & $\begin{array}{l}\text { pas du tout d'accord } 12345 \text { tout à fait } \\
\text { d'accord }\end{array}$ \\
\hline A être en harmonie avec eux & $\begin{array}{l}\text { pas du tout d'accord } 12345 \text { tout à fait } \\
\text { d'accord }\end{array}$ \\
\hline $\begin{array}{l}\text { A imposer des } \text { méthodes } \\
\text { d'organisation }\end{array}$ & $\begin{array}{l}\text { pas du tout d'accord } 12345 \text { tout à fait } \\
\text { d'accord }\end{array}$ \\
\hline Autres (précisez) & \\
\hline
\end{tabular}


II- les bonnes pratiques de management

Question 4 : Veuillez énumérez les pratiques de management qui vous semblent indispensables à la réussite d'un manager :

Question 5 : Selon vous, dans l'exécution de son travail, un salarié doit : (veuillez entourer le chiffre correspondant à votre choix)

\begin{tabular}{|l|l|}
\hline être contrôler dans son travail & pas du tout d'accord 12345 tout à fait d'accord \\
\hline se contrôler lui même & pas du tout d'accord 12345 tout à fait d'accord \\
\hline avoir des objectifs personnels & pas du tout d'accord 12345 tout à fait d'accord \\
\hline avoir des objectifs collectifs & pas du tout d'accord 12345 tout à fait d'accord \\
\hline respecter les normes de travail & pas du tout d'accord 12345 tout à fait d'accord \\
\hline apprécier son travail & pas du tout d'accord 12345 tout à fait d'accord \\
\hline Autres (précisez)
\end{tabular}

Question 6: Selon vous, une entreprise doit être organisée : (veuillez entourer le chiffre correspondant à votre choix)

selon des principes hiérarchiques

selon des procédures de travail précises

de manière souple et flexible

comme est organisée une famille

de manière harmonieuse et équilibrée

grâce à l'autorité de ses managers

Autres (précisez)

pas du tout d'accord 12345 tout à fait d'accord

pas du tout d'accord 12345 tout à fait d'accord pas du tout d'accord 12345 tout à fait d'accord pas du tout d'accord 12345 tout à fait d'accord pas du tout d'accord 12345 tout à fait d'accord pas du tout d'accord 12345 tout à fait d'accord

III- les pré-requis du bon manager

Question 7: $\quad$ la réussite d'un manager est fortement dépendante de (veuillez entourer le chiffre correspondant à votre choix) De ses diplômes et de sa formation

\begin{tabular}{|l|l|}
\hline De son réseau social & pas du tout d'accord 12345 tout à fait d'accord \\
\hline Decord
\end{tabular}

De ses connaissances techniques

De ses efforts

De son réseau familial

De ses qualités relationnelles

Autres (précisez) pas du tout d'accord 12345 tout à fait d'accord pas du tout d'accord 12345 tout à fait d'accord pas du tout d'accord 12345 tout à fait d'accord pas du tout d'accord 12345 tout à fait d'accord

Question 8 : Quelles sont les qualités de doit détenir un bon manager ? (veuillez entourer le chiffre correspondant à votre choix)

\begin{tabular}{|l|l|}
\hline la confiance en soi & pas du tout d'accord 12345 tout à fait d'accord \\
\hline La patience & pas du tout d'accord 12345 tout à fait d'accord \\
\hline le respect des ordres et de la hiérarchie & pas du tout d'accord 12345 tout à fait d'accord \\
\hline le respect de la parole des ancêtres & pas du tout d'accord 12345 tout à fait d'accord \\
\hline La sympathie & pas du tout d'accord 12345 tout à fait d'accord \\
\hline Autres (précisez) & \\
\hline
\end{tabular}

Question 9: un bon manager est avant tout (veuillez entourer le chiffre correspondant à votre choix)

\begin{tabular}{l} 
Question 9: un bon manager est avant tout (veuillez entourer le chiffre correspondant à votre choix) \\
\begin{tabular}{|l|l|}
\hline efficace & pas du tout d'accord 12345 tout à fait d'accord \\
\hline autoritaire & pas du tout d'accord 12345 tout à fait d'accord \\
\hline respectueux des valeurs traditionnelles du peuple chinois & pas du tout d'accord 12345 tout à fait d'accord \\
\hline un exemple pour les salariés & pas du tout d'accord 12345 tout à fait d'accord \\
\hline respectueux des procédures & pas du tout d'accord 12345 tout à fait d'accord \\
\hline honnête & pas du tout d'accord 12345 tout à fait d'accord \\
\hline Autres (précisez)
\end{tabular} \\
\hline
\end{tabular}


IV- les bonnes attitudes professionnelles des managers

Question 10 : Selon vous, une bonne décision managériale doit avant tout : (veuillez entourer le chiffre correspondant à votre choix)

\begin{tabular}{|l|l|}
\hline respecter l'esprit des ancêtres & pas du tout d'accord 12345 tout à fait d'accord \\
\hline respecter le cycle de la nature et des saisons & pas du tout d'accord 12345 tout à fait d'accord \\
\hline rechercher l'harmonie dans l'entreprise & pas du tout d'accord 12345 tout à fait d'accord \\
\hline se réaliser à des moments propices & pas du tout d'accord 12345 tout à fait d'accord \\
\hline être équilibrée & pas du tout d'accord 12345 tout à fait d'accord \\
\hline Autres (précisez) & \\
\hline
\end{tabular}

Question 11 : Veuillez énumérez les principales compétences que doit détenir un bon manager :

Question 12 : Quels sont les attitudes d'un manager qui vous paraissent les plus préjudiciables pour lui et son entreprise : (veuillez entourer le chiffre correspondant à votre choix)

\begin{tabular}{|l|l|}
\hline perdre la confiance de son employeur & pas du tout d'accord 12345 tout à fait d'accord \\
\hline perdre la confiance de ses subordonnés & pas du tout d'accord 12345 tout à fait d'accord \\
\hline ne pas respecter la hiérarchie & pas du tout d'accord 12345 tout à fait d'accord \\
\hline déshonorer sa famille ou ses ancêtres & pas du tout d'accord 12345 tout à fait d'accord \\
\hline ne pas respecter les procédures de travail & pas du tout d'accord 12345 tout à fait d'accord \\
\hline perdre la face & pas du tout d'accord 12345 tout à fait d'accord \\
\hline Manquer d'humilité et de retenue & pas du tout d'accord 12345 tout à fait d'accord \\
\hline Autres (précisez) & \\
\hline
\end{tabular}

\section{V-Identification}

Vous êtes :

(veuillez cocher la case correspondant à votre situation)

\begin{tabular}{|l|l|}
\hline & Une femme \\
\hline & Un homme \\
\hline
\end{tabular}

Vous avez :

(veuillez cocher la case correspondant à votre situation)

\begin{tabular}{|l|l|}
\hline & Moins de 30 ans \\
\hline & Entre 30 et 40 ans \\
\hline & Entre 40 et 50 ans \\
\hline & Entre 50 et 60 ans \\
\hline & Plus de 60 ans \\
\hline
\end{tabular}

Actuellement vous occupez des fonctions :

(veuillez cocher la ou les cases correspondant à votre situation)

\begin{tabular}{|l|l|}
\hline & Responsable de service \\
\hline & Cadre financier et administratif \\
\hline & Cadre de Production \\
\hline & Cadre commercial \\
\hline & Cadres RH \\
\hline & Autres cadres \\
\hline Autres (précisez) \\
\hline
\end{tabular}


Vous êtes :

(veuillez cocher la ou les cases correspondant à votre situation)

\begin{tabular}{|l|l|}
\hline & bouddhiste \\
\hline & confucianiste \\
\hline & taoïste \\
\hline & Non pratiquant \\
\hline Autres (précisez) \\
\end{tabular}

